Arthroskopie $2017 \cdot 30: 83-84$

DOI 10.1007/s00142-017-0137-y

Online publiziert: 3. Mai 2017

(c) Springer Medizin Verlag GmbH 2017

CrossMark

\author{
Romain Seil ${ }^{1,2} \cdot$ Wolfgang Hackl \\ 'Centre d'Orthopédie et de Médecine du Sport, Centre Hospitalier de Luxembourg, Akademisches \\ Lehrkrankenhaus der Universitätskliniken des Saarlandes, Luxembourg, Luxemburg \\ ${ }^{2}$ Sports Medicine Research Laboratory, Public Research Centre for Health, Luxembourg, Luxemburg \\ ${ }^{3}$ Universitätsklinik für Orthopädie, Medizinische Universität Innsbruck, Innsbruck, Österreich
}

\title{
Neues in der Meniskuschirurgie
}

Die vorliegende Ausgabe der Arthroskopie verdeutlicht einmal mehr, mit welch rasanten Schritten unsere Fachdisziplin sich weiterentwickelt. Insbesondere im Bereich der Meniskuschirurgie hat sich in den letzten Jahren viel getan. Stellten wir uns vor wenigen Jahren noch die Frage, ob eine Reparation des Meniskus gerechtfertigt erscheint, scheinen heute nur noch die wenigsten daran zu zweifeln [3]. Aus diesem Grund reichen die Arbeiten in diesem Heft vom Meniskuserhalt über die Arthroseprotektion bis hin zur Meniskustransplantation.

Im Rahmen der Weiterentwicklung des Meniskuserhalts sind in den vergangenen Jahren 2 Läsionstypen besondere Aufmerksamkeit gewidmet worden: den Wurzelläsionen und den Läsionen der sog. Meniskusrampe. Beide treten häufig im Zusammenhang mit Verletzungen des vorderen Kreuzbands (VKB) auf. Ausrisse der meniskotibialen Insertion des lateralen Meniskus und Läsionen der Rampe des medialen Meniskus wurden bei bis zu 15 bzw. $23 \%$ der VKBVerletzungen registriert [2,4]. Bei beiden wurden biomechanische Konsequenzen in vitro nachgewiesen, und sie werden als mögliche intraartikuläre Faktoren einer Rotationsinstabilität in Erwägung gezogen. Demnach verdichten sich die wissenschaftlichen Hinweise dahingehend, dass es sinnvoll ist, diese im Rahmen der VKB-Ersatzplastik systematisch aufzusuchen und im Sinne einer Restitutio ad integrum des verletzten Kniegelenks zu reparieren. Der Arthroskopeur wird hierbei sowohl im Rahmen der Diagnostik als auch der Therapie vor neue Herausforderungen gestellt. Beide Läsionsarten sind präoperativ nicht leicht zu diagnostizieren und intraoperativ schwer zu behandeln. Unsere Aufgabe der kommenden Jahre wird es sein, dies zu meistern, indem wir die Diagnostik verbessern, die Operationstechniken vereinfachen und ihren Nutzen wissenschaftlich absichern.

Eine weitere Domäne, der in den vergangenen Jahren nur wenig Beachtung geschenkt wurde, ist die isolierte Instabilität des Hinterhorns des lateralen Meniskus. Hierbei handelt es sich um ein seltenes und schwer zu diagnostizierendes Krankheitsbild, das posterolaterale Knieschmerzen und Blockaden bei Kniebeugung erzeugen kann. Neuere anatomische Kenntnisse haben zum besseren Verständnis dieser Entität beigetragen. Wie bei den o. g. Läsionen gilt es aber auch hier, die allgemeine Datenlage in Bezug auf Diagnostik und Therapie zu verbessern.

\section{》) Durch Meniskusersatz kann der degenerative Prozess am benachbarten Knorpel deutlich vermindert werden}

Als weiterer Aspekt soll der Ersatz von entferntem Meniskusgewebe dargestellt werden. Klinische Studien haben eindeutig gezeigt, dass durch entsprechenden Ersatz der degenerative Prozess am benachbarten Knorpel deutlich vermindert werden kann. Je nach Ausmaß des verbliebenen Meniskusrestes ist entweder ein Ersatz durch gezüchtetes Gewebe oder durch ein Allograft im Sinne einer Meniskustransplantation möglich.

Im letzten Jahrzehnt hat die Behandlung degenerativer Meniskusläsionen weltweit für viel Diskussionsstoff gesorgt.
Aus diesem Grund hat die Europäische Gesellschaft für Kniechirurgie, Sporttraumatologie und Arthroskopie (ESSKA, www.esska.org), unter der Leitung von Prof. Philippe Beaufils (Versailles, Frankreich) und Prof. Roland Becker (Brandenburg) und in Zusammenarbeit mit Mitgliedern vieler nationaler Fachgesellschaften aus 23 Ländern, eine einzigartige europäische Konsensusinitiative ins Leben gerufen. Die Originalarbeit wurde im Fachjournal der ESSKA veröffentlicht [1] und wird nun in der vorliegenden Ausgabe der Zeitschrift Arthroskopie noch einmal abgedruckt. Sie verdeutlicht den internationalen Trend zu einer zunehmenden Zurückhaltung der arthroskopischen Versorgung dieser Läsionen.

Wir bedanken uns herzlich bei den Autoren für ihre Beteiligung und hoffen, dem Leser ein interessantes Heft zu präsentieren.

Luxemburg und Innsbruck, April 2017

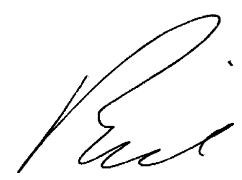

Prof. Dr. Romain Seil

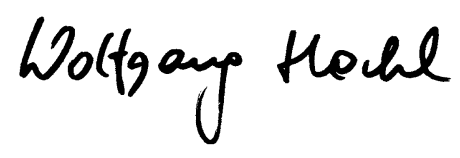

Prof. Dr. Wolfgang Hackl 


\section{Korrespondenzadresse}

\section{Prof. Dr. med. R. Seil}

Centre d'Orthopédie et de Médecine du Sport, Centre Hospitalier de Luxembourg, Akademisches Lehrkrankenhaus der Universitätskliniken des Saarlandes 78, rue d'Eich, 1460 Luxembourg, Luxemburg seil.romain@chl.lu

Interessenkonflikt. R. Seil und W. Hackl geben an, dass kein Interessenkonflikt besteht.

\section{Literatur}

1. Beaufils $P$, Becker R, Kopf $S$, Englund $M$, Verdonk $R$, Ollivier M, Seil R (2017) Surgical management of degenerative meniscus lesions: the 2016 ESSKA meniscus consensus. Knee Surg Sports Traumatol Arthrosc 25(2):335-346

2. Forkel P, Reuter S, Sprenker F, Achtnich A, Herbst E, Imhoff A, Petersen W (2015) Different patterns of lateral meniscus root tears in $A C L$ injuries: application of a differentiated classification system. Knee Surg Sports Traumatol Arthrosc 23(1):112-118

3. Seil R, Becker R (2016) Time for a paradigm change in meniscal repair: save the meniscus! Knee Surg Sports Traumatol Arthrosc 24(5):1421-1423

4. Sonnery-Cottet B, Conteduca J, Thaunat M, Gunepin FX, Seil R (2014) Hidden lesions of the posteriorhorn of the medialmeniscus:asystematic arthroscopic exploration of the concealed portion of the knee. Am JSports Med 42(4):921-926

\section{国}

\section{SpringerMedizin.de} Lesen Sie Ihre Fachzeitschrift auch als ePaper!

Als Abonnent können Sie Ihre Zeitschrift in verschiedenen Formaten lesen. Wählen Sie je nach Vorliebe und Situation aus, ob Sie die Zeitschrift als Print-Ausgabe, in Form von einzelnen Beiträgen auf springermedizin.de oder aber als komplette, elektronische ePaper-Ausgabe lesen möchten.

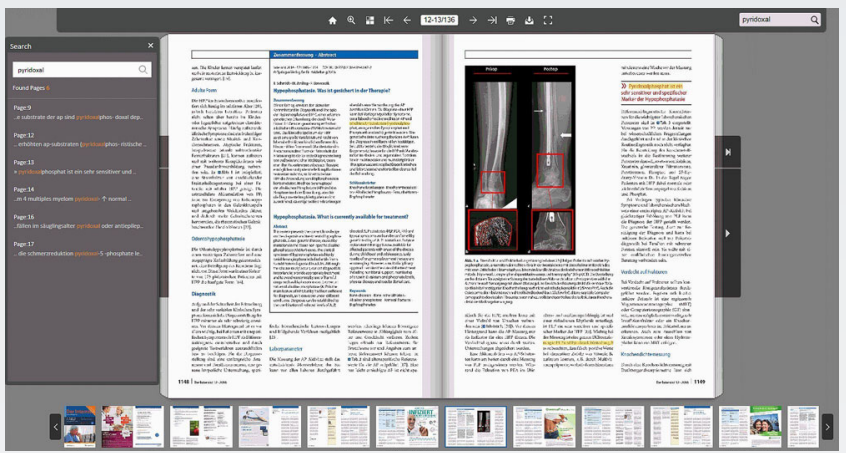

Die ePaper sind die identische Form der gedruckten Ausgaben. Sie sind nutzbar auf verschiedenen Endgeräten wie PC, Tablet oder Smartphone

Das sind die Vorteile des ePapers:

$>$ Das verlinkte Inhaltsverzeichnis führt Sie direkt zum gewünschten Beitrag.

> Eine Suchfunktion ermöglicht das Auffinden von Schlagworten innerhalb der Zeitschrift.

$>$ Jede Ausgabe kann als PDF heruntergeladen und damit auch offline gelesen werden bzw. auch gespeichert oder ausgedruckt werden.

> Als Abonnent haben Sie Zugang zu allen ePaper-Ausgaben ab 2016.

Sie finden die ePaper auf SpringerMedizin.de bei der jeweiligen Ausgabe Ihrer Fachzeitschrift. Klicken Sie auf den Button „Zum ePaper”. 